\title{
"Muss leider absagen. Muss noch nen referat fertig machen." - Zur Dialogizität von Absagen und Verabredungsablehnungen in der SMS-Kommunikation*
}

\author{
Katharina König (Münster)
}

\begin{abstract}
Short messages are used widely for the micro-coordination of appointments and meetings among friends - which also entails managing cancellations in writing. Based on a corpus of 63 text message dialogues, the paper sets out to describe different patterns: Rejections of invitations ("Verabredungsablehnungen") are distinguishable from cancellations of preconfirmed appointments ("Absagen") in their turn-internal composition. Following a dialogic approach to text messaging, the author also discusses if and in how far the conversation-analytic notion of "preference" can be applied to quasi-synchronous written dialogues. It is argued that rejections of invitations show certain features of dispreference (e.g. delay, hedging, good news exits); cancellations of pre-confirmed appointments, however, lack some of these features, as the point of reference (i.e. the day and time of the appointment) has to be re-established first.
\end{abstract}

\section{$1 \quad$ Nutzungskontexte von SMS-Kommunikation}

Die zunehmende Verbreitung von Mobiltelefonen und von den damit verbundenen Kommunikationsformen des Handytelefonats und der SMS haben nachhaltig darauf Einfluss genommen, wie HandynutzerInnen ihren Alltag organisieren und ihre sozialen Kontakte koordinieren (cf. Ling 2004; Pöschl/Döring 2012). Auch wenn SMS-Kommunikation mittlerweile in berufliche und institutionelle Kontexte Einzug gehalten hat, ${ }^{1}$ zeigen verschiedene Studien zur Nutzung des Mobiltelefons doch, dass der überwiegende Teil von SMSInteraktionen im privaten Umfeld situiert ist (cf. etwa Döring 2002; Thurlow/Poff 2013) und oft zur Bestätigung der sozialen Beziehungen, also zu einer überwiegend phatischen Kommunikation (cf. Pöschl/Döring 2012: 286f.), genutzt wird. Verabredungen und die Koordination von Terminen sind dabei die häufigsten Kommunikationsanlässe in der SMS-Kommunikation (Döring 2002: 123; Krause/Schwitters 2002: 20; Nowotny 2005: 28f.). Während man bei der Festnetztelefonie lediglich einen Ort "anwählt" und eventuell noch nach dem gewünschten

\footnotetext{
* Der vorliegende Artikel basiert auf einem Vortrag im Rahmen der Sektion "Gesprächsforschung" bei der GALSektionentagung im September 2013. Für hilfreiche Kommentare und Anmerkungen danke ich den SektionsteilnehmerInnen, Susanne Günthner, Beate Weidner und Lars Wegner.

${ }^{1}$ Einen institutionellen Rahmen für die SMS-Kommunikation bildet etwa die Politik: Angela Merkel gilt als passionierte SMS-Schreiberin, aber auch im Wahlkampf von Präsident Obama wurden SMS-Nachrichten strategisch eingesetzt (cf. Thurlow/Poff 2013).
}

Linguistik online 70, 1/15 - http://dx.doi.org/10.13092/lo.70.1747

CC by 3.0 
Gesprächspartner fragen muss, erreicht eine SMS oder ein Handyanruf die Person in der Regel direkt und persönlich (cf. Ling/Baron 2013; siehe auch Pöschl/Döring $2012 \mathrm{zu}$ "mobiler Erreichbarkeit"). Termine und Verabredungen können somit unabhängig vom Standort der adressierten Person in verschiedenen Schritten vereinbart, verschoben oder abgesagt werden. Dabei zeigt sich, dass vor allem kurzfristige terminliche Abstimmungsprozesse über das Handy abgewickelt werden (cf. Ling/Baron 2013 zum Aspekt der "micro-coordination"). Verabredungen werden somit stark auf die jeweiligen Situation und die aktuellen Aktivitäten der Interagierenden abgestimmt (cf. Nowotny 2005: 36f.) und werden häufig erst "in letzter Minute" verbindlich getroffen. Somit kann es aber auch zu kurzfristigen Absagen per SMS kommen.

Wird SMS-Kommunikation also vornehmlich zur Herstellung von sozialer Nähe und der Aufrechterhaltung des sozialen Netzwerks genutzt, so stellen missglückte Terminkoordinationen wie Ablehnungen eines Verabredungsvorschlags oder gar Absagen eine im Sinne von Brown/Levinson (1987) potenziell gesichtsbedrohende sprachliche Handlungen dar. ${ }^{2}$ Stilratgeber halten etwa fest:

Absagen können Sprengsätze sein: Sie können andere verletzten, zum Streit führen oder mit Geringschätzung gleichgesetzt werden. Viele Menschen drücken sich daher vor dieser unangenehmen Aufgabe, warten bis zum letzten Moment oder behelfen sich mit einer Notlüge [...]. (Quelle: http://www.stil.de/knigge-thema-der-woche/details/artikel/der-aktuelle-absageknigge-wie-sie-private-einladungen-und-verpflichtungen-taktvoll-absagen.html, letzter Zugriff am 21.04.2015)

Im Hinblick auf solche möglichen negativen Konsequenzen wird von der Wahl der Kommunikationsform SMS sogar explizit abgeraten: "Bei Anruf Absage: Wer ein Treffen nicht einhalten kann, sollte die Absage nicht per SMS mitteilen. Das gilt als unhöflich. Hier gilt die Devise: Lieber anrufen." (Botica 2012) Dem Übermitteln einer Absage per SMS wird somit der Status einer "schlechten Nachricht" zugeschrieben, die - so auch Maynard (2003: 249) - am besten mündlich vorgebracht werden sollte. Einzelne Studien zur SMS-Kommunikation weisen jedoch darauf hin, dass SMS in solchen Situationen auch strategisch genutzt werden können, um sich möglichen negativen Reaktionen des Gegenübers in mündlichen Interaktionen zu entziehen (cf. Dürscheid 2007: 100; Nowotny 2005: 26; Schmidt 2006: 325). ${ }^{3}$ Für die hier untersuchten missglückten Terminkoordinationen per SMS (im Folgenden als "Verabredungsablehnungen" und "Absagen" differenziert, siehe Abschnitt 3) wird entsprechend zu fragen sein, wie die SchreiberInnen das Überbringen von solchen potenziell gesichtsbedrohenden schlechten Nachrichten in quasi-synchronen schriftlichen SMS-Dialogen realisieren.

\section{Zur Dialogizität von SMS-Interaktionen}

Im linguistischen Zugriff auf SMS-Interaktionen als eine spezifische Kommunikationsform der "keyboard-to-screen communication" (Jucker/Dürscheid 2012) wurde in den vergangenen

\footnotetext{
2 Cf. Kiesendahl (2011: 130): "Bei Absagen im Allgemeinen [...] handelt es sich um eine nichtpräferierte sprachliche Handlung. Diese erfordert, dem Prinzip der Präferenzorganisation zufolge, einen kommunikativen Mehraufwand, d.h. die Absage muss in der Regel begründet werden."

${ }^{3}$ Siehe auch Ling (2008: 161): "Texting has also developed into a channel where some groups feel comfortable expressing thoughts and feelings that were once reserved for co-present interaction."
}

ISSN 1615-3014 
Jahren eine Veränderung in der Konzeptionalisierung des Gegenstandsbereichs vollzogen. In der Anfangszeit linguistischer Forschung zu sprachlichen Besonderheiten in der SMS-Kommunikation befassten sich zahlreiche Arbeiten vor allem mit orthographischen und graphostilistischen Merkmalen (cf. etwa Moraldo 2002; Schlobinski 2005; Schlobinski et al. 2001). ${ }^{4}$ Ebenso stand die Frage danach, wie die SchreiberInnen mit der Beschränkung auf 160 Zeichen pro Nachricht umgehen, im Mittelpunkt des linguistischen Interesses (cf. etwa Dittmann 2006 zu syntaktischen Reduktionen und Abkürzungen bei SMS-Nachrichten). Viele dieser frühen Untersuchungen zur "SMS-Sprache" hatten somit vor allem einzelne SMS-Nachrichten zum Gegenstand; wie sich jedoch die Einbindung einzelner Nachrichten in eine Abfolge von Nachrichten bzw. in eine spezifische SMS-Gattung auf die sprachliche Gestaltung auswirkt, wurde nur selten in den Blick genommen (siehe auch die Kritik bei Spagnolli/Gamberini 2007: 345, König/Bahlo 2014).

Weitere Arbeiten zeigen jedoch, dass Gruppen von SchreiberInnen ihre jeweils eigenen Schreibstrategien entwickeln (cf. Androutsopoulos/Schmidt 2004), dass sich verschiedene SMS-Gattungen herausgebildet haben (cf. Androutsopoulos/Schmidt 2002; Hauptstock/König/Zhu 2010; Imo 2012; Ling/Julsrud/Yttri 2005) und dass die Anordnung einzelner Nachrichten zu einem zusammenhängenden Dialog auf die Wahl der sprachlichen Mittel in einer SMS-Nachricht Einfluss hat (etwa: Weglassen von Anreden und Begrüßungen in einem fortlaufenden Dialog, cf. Günthner 2011). SMS-Interaktionen werden solchen Ansätzen gemäß als "getippte Dialoge" (Dürscheid/Brommer 2009) oder "adjazente Quasi-Dialoge" (Dittmann 2006: 84) klassifiziert (cf. auch Schwitalla 2002). Wie diese Dialogizität der SMSKommunikation in einer konversationsanalytischen Herangehensweise konzeptionalisiert werden kann, wird ausführlich bei Günthner $(2011 ; 2012)$ herausgearbeitet. $^{5}$

SMS-Nachrichten stellen [... keine monologischen, interaktionslosgelösten Kommunikationsformen dar, sondern sie richten sich - selbst wenn sie nicht in eine Face-to-faceSituation eingebettet sind, sondern der Rezipient räumlich entfernt ist und zeitlich verzögert reagiert - dialogisch an einem Gegenüber aus: Sie nehmen häufig Bezug auf vorherige kommunikative Handlungen des Gegenübers und machen Folgehandlungen erwartbar. (Günthner 2011: 7)

Auch für schriftliche SMS-Interaktionen lässt sich also eine geordnete und aufeinander bezogene Abfolge verschiedener sprachlicher Handlungen beschreiben; es finden sich Adjazenzpaare, bei denen der jeweils erste Paarteil (first pair part) die Einlösung eines zweiten Paarteils (second pair part) durch das Gegenüber konditionell relevant macht (cf. auch Hauptstock/König/Zhu 2010). Dabei richten sich die SMS-SchreiberInnen bei der Gestaltung ihrer Beiträge an geteilten bzw. in der SMS-Interaktion hervorgebrachten Wissensbeständen aus (zu Aspekten des "recipient design" bei SMS siehe Günthner 2011). Ebenso zeigt sich die dialogische Rahmung von SMS-Interaktionen an der Etablierung einer "rituellen Klammer" mit Anreden, die meist nur zu Beginn eines SMS-Dialogs realisiert werden (cf. auch Bernicot et al. 2012), und Beendigungssignalen zum Abschluss der Dialogstrukturen (cf. Spilioti 2011). Auch

\footnotetext{
4 Ähnlich lautet auch der Befund für die linguistische Analyse computervermittelter Kommunikation generell: "Those early days were also characterized by a fascination with superficial structural features, such as acronyms, abbreviations, and emoticons [...]" (Herring/Stein/Virtanen 2013: 3).

${ }^{5}$ Cf. auch Günthner/Kriese (2012) zu einer deutsch-chinesisch kontrastiven Untersuchung zu Dialogizität in der SMS-Kommunikation.
} 
wenn Produktion und Rezeption von einzelnen SMS-Nachrichten nicht gleichzeitig ablaufen und die Kommunikationsform der SMS daher als asynchron bis quasi-synchron eingestuft werden muss (cf. Dürscheid 2002; 2007), zeigen die Arbeiten von Günthner (2011; 2012) auf, dass sich grundlegende Konzepte der Konversationsanalyse auf den dialogischen und aufeinander bezogenen Austausch von SMS übertragen lassen.

Die vorliegende Untersuchung ergänzt bisherige konversationsanalytisch geprägte Arbeiten zu Dialogizitätsmerkmalen in SMS-Interaktionen um eine Betrachtung der Prinzipien und Merkmale von Präferenzorganisation (cf. Pomerantz/Heritage 2013; Schegloff 2007: 58-96) in SMS-Dialogen. In der strukturellen Markierung von Beiträgen als präferiert oder dispräferiert drücken sich zwar keine individuellen psychologischen Präferenzen aus, jedoch können soziale Normen in diesem Mechanismus kodiert sein (cf. Lerner 1996). So erläutert etwa Heritage (1984: 268) für die Präferenzorganisation u.a. bei Einladungen in mündlichen Interaktionen: "It will be obvious enough that the preferred format responses to $[\ldots]$ invitations $[\ldots]$ are uniformly affiliative actions which are supportive of social solidarity, while dispreferred format responses are largely destructive of social solidarity." Heritage vertritt hier also die Auffassung, dass solche sprachlichen Handlungen, die die soziale Beziehung zwischen den Interagierenden gefährden in der Regel in einem dispräferierten Format realisiert werden. ${ }^{6}$

Wurde für mündliche Interaktionen vor allem das zeitlich verzögerte Einsetzen eines zweiten Beitrags als Möglichkeit zur Markierung von Dispräferenz beschrieben (cf. etwa Davidson 1984; Pomerantz 1984; Schegloff 2007: 63-73), so ist eine einfache Übertragung dieser "reluctance markers" (Bilmes 1988) auf die SMS-Kommunikation problematisch, da der zeitliche Abstand, ab wann eine Antwort als verzögert positioniert gilt, von gruppenspezifischen Erwartungshaltungen abhängen kann (siehe etwa Laursen 2005) und die SchreiberInnen nie ausschließen können, dass auch andere Faktoren zu einer Verzögerung geführt haben. Wenn also rein zeitliche Verzögerungen bis zum Übermitteln der nächsten Nachricht nicht immer konklusiv zur Markierung von Dispräferenz dienen können, so soll im Folgenden die Frage gestellt werden, mit welchen anderen Mitteln die SchreiberInnen die Dispräferenz einer durch SMS vermittelten sprachlichen Handlung signalisieren.

\section{Absagen und Verabredungsablehnungen}

Unter dem Begriff "Absage" werden in linguistischen Studien verschiedene kommunikative Aktivitäten gefasst. So untersuchen etwa Schindler (2004) und Drescher (1994) schriftliche Absagen, die auf eine Bewerbung auf eine ausgeschriebene Stelle oder die Bewerbung um einen Studienplatz geschickt werden. Die Absagebriefe werden in den beiden Studien als reaktive sprachliche Handlungen klassifiziert, da die Briefe auf eine vorher eingegangene Bewerbung Bezug nehmen ("die Absage als 'dialogisch rückwärts verweisender Brief' [...], d. h. sie ist abschließender Teil eines Briefwechsels", Drescher 1994: 120f.; "Absage als zweiten Zug des Verwaltungsprozesses", Schindler 2004: 231). Spezifisch mit Absagen als Mitteilungen darüber, dass ein zuvor vereinbarter Termin nicht eingehalten werden kann, die auch Gegenstand des vorliegenden Beitrags sind, befasst sich Wagner (2001). Auch bei Wagner findet sich die Idee, dass diese Absagen als "reaktiver Sprechakt" zu klassifizieren sind, da sie

\footnotetext{
${ }^{6}$ Ausnahmen bilden hier etwa Streitinteraktionen, in denen das Präferenzsystem umgekehrt wird und direkter Widerspruch im präferierten Format realisiert wird (cf. Kotthoff 1993).
} 
sich auf die vorher bereits vollzogenen Terminvereinbarung zurückbeziehen: "Der Sprecher setzt diejenigen Selbst- und Fremdverpflichtungen außer Kraft, die sich für ihn und für den Hörer aus einer vorausgegangenen Vereinbarung ergeben haben [...]." (Wagner 2001: 170)

Die Annahme, dass Absagen stets als reaktiver Zug zu konzeptionalisieren sind, wird jedoch nicht in allen linguistischen Studien geteilt: So unterschiedet etwa Kiesendahl (2011) bei misslungenen Terminkoordinierungen per E-Mail zwischen "Ablehnungen" und "Absagen". Während bei Absagen "Sprecher und Adressat im Vorfeld bereits eine Absprache/Vereinbarung getroffen haben, die der Sprecher nun revidieren möchte" (Kiesendahl 2011: 123), finden Terminablehnungen in Kontexten statt, in denen die SprecherInnen zuvor kein Treffen vereinbart haben (siehe auch Kiesendahl 2011: 336). Dies bedingt nun, dass Absagen und Terminablehnungen an unterschiedlichen sequentiellen Positionen realisiert werden: "Der Unterschied existiert dahingehend, dass [ABLEHN] eine reaktive Sprechhandlung ist, während [ABSAG] stets initiativen Charakter hat und eine im Vorhinein bereits getroffene Absprache auflöst." (Kiesendahl 2011: 124) Absagen wird bei Kiesendahl (2011) also initiativer Handlungscharakter zugeschrieben, während Drescher (1994), Schindler (2004) und Wagner (2001) Absagen als Abschluss eines Dialogs fassen. In dem vorliegenden Beitrag, der Kiesendahls Unterscheidung zwischen Ablehnungen von vorausgehenden Terminvorschlägen und Absagen von bereits vereinbarten Terminen übernimmt, wird zu fragen sein, wie Absagen bzw. Verabredungsablehnungen sequentiell in SMS-Dialoge eingebunden werden bzw. inwieweit Absagen in dieser Kommunikationsform als initiativ oder reaktiv beschrieben werden können.

Des Weiteren soll in einem Abgleich von Verabredungsablehnungen und Absagen der Frage nachgegangen werden, ob diese potenziell gesichtsbedrohenden sprachlichen Handlungen auf der sprachlichen Oberfläche als "dispräferiert" markiert werden. Im Rahmen der Analyse soll diskutiert werden, ob und wie sich das konversationsanalytische Konzept der "Präferenz" (cf. Pomerantz/Heritage 2013; Schegloff 2007: 58-96), das spezifisch für gesprochensprachliche, in der Zeit verlaufende Interaktionen mit ko-präsenten Interagierenden entwickelt wurde, auf die medial schriftliche und asynchrone bis quasi-synchrone dialogische SMS-Kommunikation anwenden lässt. Hier wird zu untersuchen sein, wie Absagen bzw. Verabredungsablehnungen intern strukturiert sind, inwiefern sich also ein verfestigter Aufbau beschreiben lässt, ${ }^{7}$ an dem eine spezifische Präferenzorganisation bei Absagen bzw. Verabredungsablehnungen ablesbar ist.

Terminabsagen werden in linguistischen Arbeiten häufig auch unter dem Begriff "Entschuldigung" gefasst (cf. etwa Davies/Merrison/Goddard 2007; Staffeldt 2013; Weidacher 2011, spezifisch für SMS siehe Schmidt 2006). Auch im Duden Universalwörterbuch findet sich für den Eintrag "Entschuldigung" neben der Bedeutung "Begründung, Rechtfertigung für einen Fehler, ein Versäumnis" die Angabe "[schriftliche] Mitteilung darüber, nicht anwesend sein, nicht teilnehmen zu können" (Duden 2003: 471). Entsprechend untersucht etwa Weidacher (2011) unter dem Begriff "Entschuldigungsmails" solche E-Mails, in denen

\footnotetext{
${ }^{7}$ Für Absagen per SMS hat Schmidt im Jahr 2006 festgehalten, dass sich die Nachrichten "im momentanen status nascendi einer kommunikativen Gattung" (Schmidt 2006: 326) befinden. Für die hier untersuchten Daten (cf. Abschnitt 4 für eine Korpusbeschreibung) wird daher die Frage gestellt, ob sich verfestigte kommunikative Muster (cf. Günthner 1995) beschreiben lassen.
} 
Studierende ihre Abwesenheit bei Lehrveranstaltungen "entschuldigen" (cf. auch Kiesendahl 2013, die zwischen auf die Vergangenheit und auf die Zukunft gerichteten Entschuldigungen unterscheidet). Weidacher stellt jedoch fest, dass nur etwa die Hälfte der von ihm untersuchten Entschuldigungsmails überhaupt eine explizite Entschuldigungsfloskel enthält (Weidacher 2011: 69). ${ }^{8}$ Daher sollen die misslungenen Terminkoordinationen per SMS im Folgenden zudem auch auf das Vorkommen und die Funktion von Entschuldigungsfloskeln (beispielsweise tut mir leid oder sorry, cf. auch Robinson 2004) untersucht werden.

\section{$4 \quad$ Verabredungsablehnungen per SMS}

Die im Folgenden untersuchten Daten sind der SMS-Datenbank am Centrum für Sprache und Interaktion (Susanne Günthner) entnommen. Die Datenbank umfasst derzeit 1747 SMSDialoge mit 7459 einzeln gesendeten Nachrichten (Stand September 2013), die überwiegend zwischen Studierenden, aber auch zwischen Studierenden und ihren Eltern, KollegInnen oder Arbeitgebern ausgetauscht wurden. Bei der Einstellung der SMS-Dialoge in die Datenbank werden neben Metadaten $\mathrm{zu}$ den SchreiberInnen (Alter, Geschlecht, Beruf) und Schreibbedingungen (Tarif, T9-Unterstützung) auch Kategorisierungen für die SMS-Dialoge eingegeben (cf. Imo 2012: 24). Es zeigt sich, dass der Kategorie "Verabredung" ein hoher Stellenwert zukommt: In immerhin 721 Dialogen sind die SchreiberInnen mit der Koordination von Terminen befasst. Die folgenden Analysen basieren auf einem Korpus von insgesamt 63 misslungenen Terminkoordinationen per SMS aus den Jahren 2010-2013. Hieraus sind 28 als Absagen eines zuvor bereits vereinbarten Termins einzustufen; 35 können dagegen als Verabredungsablehnungen klassifiziert werden, da in ihnen ein Verabredungsvorschlag negativ beantwortet wird.

Die Definition von "Verabredungsablehnungen" als Ablehnungen eines zuvor hervorgebrachten Verabredungsvorschlags impliziert, dass es sich hierbei um ein reaktives Format handelt (Kiesendahl 2011); Verabredungsablehnungen per SMS können also als second pair part eines Adjazenzpaares eingestuft werden (siehe etwa Schegloff 2007: 13-21). Auch wenn denkbar ist, dass die eigentliche Verabredungseinladung in einem face-to-face-Gespräch oder einem Telefonat hervorgebracht wird, ist der Verabredungsvorschlag als first pair part in den hier untersuchten Daten in allen Fällen per SMS realisiert. Bei der Ablehnung dieser Terminvorschläge lassen sich nun verschiedene Muster identifizieren, die im Folgenden anhand einzelner Beispiele vorgestellt werden sollen.

\subsection{Muster (1): Begründung der Ablehnung vor expliziter Ablehnung}

In dem folgenden SMS-Dialog wendet sich Schreiberin A mit dem Vorschlag, gemeinsam laufen zu gehen, an ihre Freundin Anna. ${ }^{9}$ Anna wiederum lehnt diesen Verabredungsvorschlag $\mathrm{ab}$ :

\footnotetext{
${ }^{8}$ Zudem kann infrage gestellt werden, ob mit Formulierungen wie "Leider muss ich mich für die heutige Einheit des PS Text und Kompetenz entschuldigen, da ich eine Magen-Darm-Grippe habe" (Beispiel A bei Weidacher 2011: 57) überhaupt Entschuldigungshandlungen zum Ausdruck kommen sollen oder ob lediglich die Nichtteilnahme mit einer Bedauernsbekundung mitgeteilt wird.

9 Alle Namens- und Ortsreferenzen in dieser und den folgenden Nachrichten sind durch Pseudonyme ersetzt worden.
} 


\section{Dialog \#456}

Hallo Anna!ich werde so um halb sechs zum Westpark
düsen und ne runde laufen!hast du Zeit mitzukommen?
Nachricht \#1 - 11.02.2011 - 16:04:00

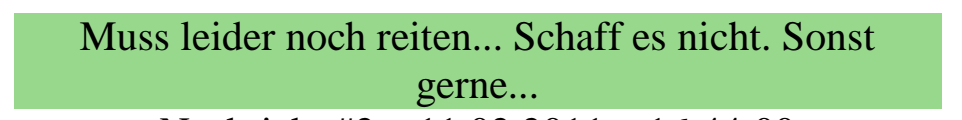

Nachricht \#2 - 11.02.2011 - 16:44:00

Sekundenangaben fiktiv ${ }^{10}$

Wie ist nun die Verabredungsablehnung intern strukturiert und aufgebaut? Eine explizite Anrede erfolgt in Nachricht \#2 nicht; stattdessen reagiert Anna in direkter Anbindung an die an sie gerichtete Anfrage (cf. Günthner (2011: 12) zum Wegfall von Anreden in SMS-Dialogen zur Kontextualisierung einer Dialogfortführung). Der Zeitpunkt der Verabredung wird nicht noch einmal explizit verbalisiert, sondern als gemeinsam geteiltes Hintergrundwissen behandelt. ${ }^{11}$ Anna beginnt die Ablehnung des Verabredungsvorschlags mit einer Begründung ("Muss leider noch reiten...", Nachricht \#2; cf. Atkinson/Drew 1979: 160-170; Buttny/Morris 2001; Scott/Lyman 1968 zu accounts), mit dem sie die daraufhin in der gleichen Nachricht verbalisierte explizite Ablehnung "Schaff es nicht." (Nachricht \#2) andeutet und für Schreiberin A nachvollziehbar macht. Das Modalverb "müssen" kommt hierbei häufig zum Einsatz, um anzuzeigen, dass die Ablehnung durch äußere Umstände erzwungen wird (cf. etwa Weinrich 2005: 300; Talmy 1988: 77-88 zu "force dynamics" bei Modalverben). Ablehnungen aufgrund von persönlichen Präferenzen (z.B. kein Interesse an der vorgeschlagenen Aktivität) kommen in den untersuchten Daten hingegen nicht vor. Die eigentliche Mitteilung der Ablehnung "Schaff es nicht." wird hier also erst an zweiter Position des reaktiven Zugs realisiert, was zu einer "Trennung" von ersten und zweitem Paarteil führt und als Dispräferenzmarker eingestuft werden kann ("break the contiguity of first and second pair part", Schegloff 2007: 67). ${ }^{12}$ Das sich nun anschließende "Sonst gerne" dokumentiert Annas prinzipielles Interesse an der von Schreiberin A vorgeschlagenen gemeinsamen Aktivität. Mit Maynard (2003) kann ein solcher Abschluss eines SMS-Dialog-Zugs als "good news exit" klassifiziert werden. ${ }^{13}$ In mündlichen Interaktionen werden überbrachte schlechte Nachrichten durch die Betonung der positiven Aspekte der Nachricht abgemildert und zugleich abgeschlossen. In diesem SMS-Dialog kommt es nach dem zweiten Zug durch Anna zu keiner weiteren Reaktion durch Schreiberin A; die Paarsequenz aus Verabredungsvorschlag und Verabredungsablehnung bildet somit einen "Minimaldialog" (cf. Schmidt 2006).

\footnotetext{
10 Bei der Einstellung der SMS-Dialoge in die Datenbank müssen immer Sekundenangaben gemacht werden, um den genauen Ablauf des Dialogs abbilden zu können. Liegen die genauen Angaben nicht vor, so sind sie als "fiktiv" gekennzeichnet.

11 Diese Nicht-Wiederholung des Verabredungszeitpunkts wird besonders im Abgleich mit den sprachlichen Verfahren in Absagen relevant (cf. Abschnitt 5).

12 Mit Schegloff (2007: 68-69) kann auch von einem "anticipatory account" gesprochen werden: "[S]uch components regularly are positioned early in the turn, where they serve as well to delay the second pair part itself and thereby (further) break the contiguity with the first pair part".

${ }^{13}$ Siehe etwa Maynard (2003: 177): "The sequelae to bad news in conversation suggest that it is impermissible to make a transition from such deliveries to other types of talk without first putting a positive face on the bad news."
} 


\subsection{Muster (2): Entschuldigung/Bedauern vor Begründung der Ablehnung}

Ein weiteres Muster, das in den untersuchten Verabredungsablehnungen durch SMS häufig vorkommt, soll anhand des nächsten Beispiels analysiert werden. In diesem Muster wird die Ablehnung nicht explizit verbalisiert, sondern lediglich durch eine Entschuldigungsfloskel oder einen Ausdruck des Bedauerns (z. B. schade) angedeutet und durch eine folgende Begründung für die DialogpartnerInnen plausibilisiert.

\section{Dialog \#528}

Hello, hast du morgen Zeit und lust zu spielen oder musst du Analysen schreiben?;-)

Nachricht \#1 - 23.03.2011 - 17:54:05

Ehm sorry habe bis viertel nach 4 schule und dann noch training. Wie siehts freitag um 4 aus? Gruß Lisa:) Nachricht \#2 - 23.03.2011 - 17:54:55

\section{Freitag passt glaub}

Nachricht \#3 - 23.03.2011 - 22:08:05

Oh zu früh abgeschickt...egal...sehen uns Freitag,lg

Nachricht \#4 - 23.03.2011 - 22:08:20

Sekundenangaben fiktiv

Die Anfrage von Schreiberin A, die bereits einen möglichen Ablehnungsgrund antizipierend in ihren Verabredungsvorschlag einbaut (oder musst du Analysen scheiben? Nachricht \#1), ${ }^{14}$ wird von Lisa nicht offen abgelehnt. Lisa deutet vielmehr durch eine informelle Entschuldigungsfloskel (sorry, Nachricht \#2) an, dass sie das vorgeschlagene Treffen nicht einrichten kann (auch hier wird der genannte Zeitpunkt nicht wiederholt, sondern als gemeinsames Hintergrundwissen behandelt). Es wird somit Schreiberin A überlassen, zu rekonstruieren, dass die Nachricht eine Ablehnung des vorherigen Vorschlags darstellt. ${ }^{15}$ Die Nachricht ist ebenfalls dadurch als dialogisch auf die Vorgängernachricht bezogen markiert, dass Lisa keine direkte Anrede anführt (cf. Günthner 2011). Auf die Entschuldigungsfloskel folgt wiederum eine Begründung, die die Ablehnung für Schreiberin A nachvollziehbar macht (hab bis viertel nach 4 schule und dann noch training, Nachricht \#2). In Anlehnung an Robinson kann hier also von einem "apology-prefaced account" (Robinson 2004: 299)

\footnotetext{
${ }^{14}$ Dies kann zum einen als Strategie gewertet werden, der adressierten Person einen gesichtsschonenden Weg der Ablehnung zu eröffnen. Nicht ausgeschlossen werden kann an dieser Stelle allerdings, dass es sich bei der Angabe "oder musst du Analysen schreiben" um eine Frotzelaktivität handelt (cf. Günthner 1999), mit der Schreiberin A auf vorherige Ablehnungen von Schreiberin B zurückverweist und diese somit sogar als möglichen Absagegrund disqualifiziert (auf den Status als Frotzelei kann etwa das angehängte zwinkernde Emoticon verweisen).

15 Cf. Drew (1984: 137): "Reportings recurrently involve a speaker detailing some activities or circumstances without explicitly stating the implications of the reporting, an upshot or consequence. [...] It is left to the recipient to discover the upshot of a reporting." Ebenso wird diese Praktik zur Übermittlung von schlechten Nachrichten bei Schegloff (1988) beschrieben.
} 
gesprochen werden; die Entschuldigung ist nicht die hauptsächliche kommunikative Handlung der SMS-Nachricht, ${ }^{16}$ sondern kontextualisiert das Folgende als Ablehnung.

Nicht nur die Tatsache, dass die Ablehnung nicht expliziert wird, sondern auch das vorgeschaltete Verzögerungssignal Ehm markierten Lisas Reaktion auf den Verabredungsvorschlag als dispräferiert. Der am Ende von Nachricht \#2 gegebene Vorschlag eines alternativen Termins kann als funktional parallel zu dem "good news exit" (Maynard 2003) in Dialog \#456 als Strategie beschrieben werden, mit der auch bei der aktuellen Ablehnung eines Kontakts das prinzipielle Interesse an weiteren Kontakten betont wird. Durch die Nennung eines Alternativtermins ergibt sich für Schreiberin A nun jedoch die konditionelle Relevanz, den Vorschlag entweder anzunehmen oder ebenfalls abzulehnen, sodass hierdurch eine Expansion des Dialogs um mindestens einen weiteren Zug erwartbar wird.

\subsection{Muster (3): Motivationsbekundung vor aber-Begründung}

Eine ähnliche Strategie der Betonung eines prinzipiellen Kontaktinteresses findet sich auch in einem dritten, häufig in den untersuchten Daten auftretenden Muster. Auch hier wird die eigentliche Ablehnung nicht explizit realisiert, sondern muss durch die Interaktionspartnerin/den Interaktionspartner rekonstruiert werden; der Aufbau der Ablehnung gestaltet sich jedoch anders als in Muster (2).

\section{Dialog \#295}

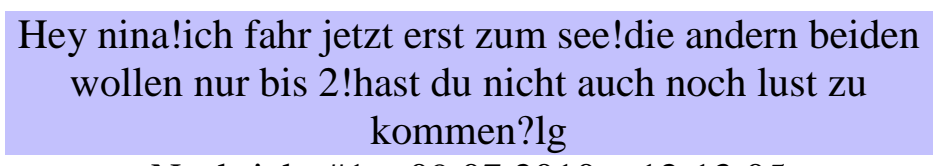

Nachricht \#1 - 09.07.2010 - 12:13:05

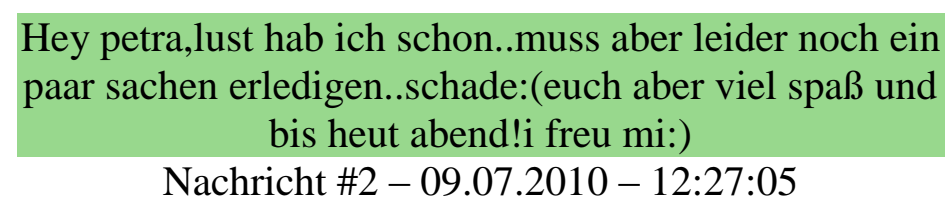

\section{Sekundenangaben fiktiv}

In ihrer Verabredungsablehnung nimmt Nina Petras Formulierung, ob sie noch Lust habe, zum See zu kommen, zunächst affirmativ auf (lust hab ich schon, Nachricht \#2) und zeigt somit ebenfalls an, dass sie ein prinzipielles Interesse an dem Kontakt zu Petra hat. Die Voranstellung des von Petra eingeführten Wortes "Lust" zeigt dabei eine direkte thematische Kohäsion zu Petras erster Nachricht. In einer durch ein adversatives "aber" angeschlossenen Begründung benennt Nina nun jedoch Faktoren, die ihr das Annehmen des Verabredungsvorschlags nicht ermöglichen. ${ }^{17}$ Hierbei fällt - wie auch schon in Dialog \#456- die Verwendung des Modalverbs "müssen" auf, das Ninas Verabredungsablehnung als extern motiviert rahmt.

\footnotetext{
16 Dies zeigt sich etwa auch an der Reaktion von Schreiberin A: Sie geht nicht noch einmal gesondert auf die Entschuldigungsfloskel ein (cf. Robinson (2004) zum Kriterium der Rezipientenreaktion).

${ }^{17}$ Auch hier wird der vorgeschlagene Zeitpunkt für ein Treffen nicht noch einmal in der Verabredungsablehnung wiederholt.
} 
Eine tatsächliche Ablehnung wird jedoch auch durch die angehängte Bedauernsbekundung schade nicht ausformuliert; Nina geht also davon aus, dass Petra aus dem bisherigen Interaktionskontext erschließen kann, dass es sich bei ihrem zweiten Zug um eine Ablehnung handelt. Im Sinne eines "good news exit" (Maynard 2003) wünscht Nina Petra und den anderen Freundinnen, die mit zum See fahren, "viel spaß", um dann auf ein anderes Treffen zu verweisen, bei dem sich Petra und Nina noch am gleichen Tag begegnen werden. Es kommt zu keiner weiteren Reaktion auf Nina zweiten Zug, sodass sich hier ebenso ein Minimaldialog aus dem Adjazenzpaar "Verabredungsvorschlag - Verabredungsablehnung" ergibt.

Auch das Verfahren, zunächst positive Aspekte hervorzuheben, bevor die eigentliche schlechte Nachricht überbracht wird, ist in der Konversationsanalyse als Möglichkeit zur Markierung von Dispräferenz beschrieben worden (cf. Pomerantz 1978: 86; Schegloff 2007: 69). Es stellt ein weiteres Vorgehen dar, mit dem ein zweiter Teil in einem SMS-Dialog erst "verspätet" realisiert und somit als "dispräferiert" markiert wird.

\subsection{Ausnahmen: Explizite Ablehnung vor Begründung der Ablehnung}

Während die eigentliche Ablehnung des Verabredungsvorschlags in den vorhergehend beschriebenen Mustern entweder "verspätet" oder gar nicht realisiert wurde, finden sich auch einige wenige Ausnahmen von diesen als dispräferiert markierten Ablehnungen. Betrachtet man etwa die folgenden zwei Züge eines Verabredungsvorschlags mit anschließender Ablehnung, so fällt - gerade in Abgleich mit den bislang untersuchten SMS-Dialogen - auf, dass die Ablehnung direkt zu Beginn des reaktiven Zugs platziert wird.

\section{Dialog \#1647 (Ausschnitt)}

Magst du noch vorbeikommen? habe salat gemacht, mit vinaigrette Bitte!

Nachricht \#6 - 20.10.2010 - 23:13:11

Heute geht nicht. muss noch aufräumen und so. sehen uns ja eh morgen dann. gute nacht!

Nachricht \#7 - 20.10.2010 -c 23:19:37

Anders als in den bisherigen Beispielen für Verabredungsablehnungen ist die Begründung in der reaktiven Nachricht erst nach einer expliziten Ablehnung positioniert. Die direkte Ablehnung des Vorschlags ist außerdem dadurch markiert, dass Schreiberin A zu Ende ihrer Anfrage durch ein Bitte! ihr Interesse an einem Treffen stark betont hat und eine Ablehnung damit in jedem Fall gesichtsbedrohend wäre.

Wie kann nun eine solche Abweichung von der bisherigen Tendenz, Ablehnungen durch ihre verspätete oder ausbleibende Realisierung als dispräferiert zu markieren, erklärt werden? Hierfür ist es notwendig, den Kontext, in den das Adjazenzpaar eingebettet ist, genauer zu betrachten. Die "direkte" Formulierung einer Ablehnung erfolgt in Zusammenhang mit einem Streitdialog, in dem Schreiberin A Schreiber B zuvor schon mehrmals beleidigt hat. 


\section{Dialog \#1647}

Weißte was? Ist mir egal! Mach doch was du willst, knutsch doch wen du willst, von mir aus verreck! du bist so unfassbar egoistisch! bah...

Nachricht \#1 - 20.10.2010 - 16:50:31

Tut mir leid... warum antwortest du nicht? :( ich war halt arg enttäuscht von dir..

Nachricht \#2 - 20.10.2010 - 20:15:44

Ein bisschen weniger hysterie wäre mir lieb.

Nachricht \#3 - 20.10.2010 - 21:03:31

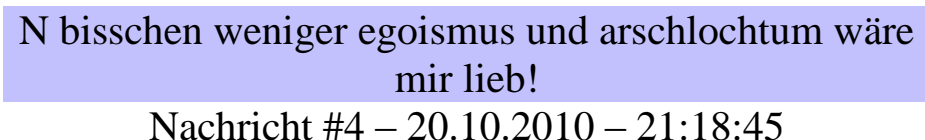

Nachricht \#4 - 20.10.2010 - 21:18:45

Tschuldigung.

Nachricht \#5 - 20.10.2010 - 21:21:10

Magst du noch vorbeikommen? habe salat gemacht, mit vinaigrette Bitte!

Nachricht \#6 - 20.10.2010 - 23:13:11

Heute geht nicht. muss noch aufräumen und so. sehen uns ja eh morgen dann. gute nacht!

Nachricht \#7 - 20.10.2010 - 23:19:37

Gute Nacht! :(

Nachricht \#8 - 20.10.2010 - 23:25:46

Sekundenangaben fiktiv

Wiederholt kritisiert Schreiberin A ihren Partner Schreiber B und macht ihm Vorwürfe, um sich dann - als eine Reaktion von Schreiber B auf diese Nachrichten jeweils ausbleibt - in einem folgenden Zug zu entschuldigen (Tut mir leid, Nachricht \#2; Tschuldigung, Nachricht \#5). Hier wird also das "Schweigen" von Schreiber B durch Schreiberin A interpretiert (cf. Laursen 2005). Als Schreiber B nach etwa zwei Stunden immer noch nicht auf die vorhergehende Entschuldigung von Schreiberin A reagiert hat, schlägt diese ein Treffen bei sich vor (Nachricht \#6). Aus diesem konfrontativen Streitkontext wird nun ersichtlich, warum die sonst angewendeten Verfahren, mit denen Ablehnungen als dispräferiert markiert werden, hier nicht zum Einsatz kommen: Die "direkte" Ablehnung von Schreiber B nimmt die zuvor bereits etablierte Streitmodalität auf und re-aktualisiert diese zugleich (cf. Kotthoff 1993). Dennoch schließt Schreiber B an seine direkte Ablehnung im Sinne eines "good news exit" (Maynard 2003) einen Verweis auf ein kommendes Treffen an (sehen uns ja eh morgen dann, Nachricht \#7) und verdeutlicht somit, dass er den Kontakt zu Schreiberin A nicht prinzipiell ablehnt.

\subsection{Zwischenfazit zu Verabredungsablehnungen per SMS}

Zusammenfassend kann für die hier untersuchten reaktiven Verabredungsablehnungen per SMS festgehalten werden, dass sie in der Regel durch verschiedene Verfahren des internen Aufbaus des ablehnenden SMS-Zugs als dispräferiert markiert sind. Entweder werden die 
Ablehnungen selbst gar nicht ausformuliert, sondern der Rekonstruktion des Dialogpartners/der Dialogpartnerin überlassen, oder die Mitteilung einer Ablehnung erfolgt erst "verspätet" im reaktiven Ablehnungszug. In diesem Fall wird eine Begründung vor der eigentlichen Ablehnung platziert. Explizite Entschuldigungsfloskeln kommen nicht in allen Verabredungsablehnungen vor. In solchen Fällen, in denen die Ablehnung nicht explizit formuliert wird, können sie aber vor der Begründung platziert werden und an dieser Position als Kontextualisierungshinweis für eine Ablehnung dienen. Dass die SchreiberInnen eine Ablehnung wie das Überbringen einer schlechten Nachricht behandeln, wird zudem an "good news exit"-Verfahren (cf. Maynard 2003) ersichtlich, mit denen die ablehnende Person verdeutlicht, dass ihre Ablehnung nicht als generelles Desinteresse an der vorgeschlagenen Aktivität oder am Kontakt mit der anfragenden Person verstanden werden soll.

Dass es sich bei Verabredungsablehnungen um einen zweiten Teil des Adjazenzpaares "Verabredungsvorschlag - Verabredungsablehnung" handelt, wird auch auf der sprachlichen Oberfläche ersichtlich. Überwiegend wird in der reaktiven Ablehnungs-SMS keine Anrede und keine Begrüßung mehr realisiert, der zweite Zug wird somit als Teil eines fortgeführten SMSDialogs realisiert. In den meisten Fällen konstituiert die Abfolge von Verabredungsvorschlag und Ablehnung einen Minimaldialog; die SMS-Dialoge enden also meist nach dem zweiten Zug. Kommt es dennoch zu einer Erweiterung um einen oder weitere Züge, so ist dies meist darauf zurückzuführen, dass die Person, die einen Verabredungsvorschlag abgelehnt hat, wiederum einen Alternativtermin vorgeschlagen hat. ${ }^{18}$

\section{$5 \quad$ Absagen per SMS}

Bei Absagen handelt es sich wie auch bei Verabredungseinladungen um potenziell gesichtsbedrohende Aktivitäten (cf. Brown/Levinson 1987); das Gesicht der absagenden Person kann bedroht sein, da beim Gegenüber der Eindruck entstehen könnte, dass die absagende Person unzuverlässig ist. Aber auch die Belange der adressierten Person sind insofern betroffen, als eine bestehende Erwartung ihrerseits - die Erwartung, dass ein Treffen zu dem vereinbarten Zeitpunkt stattfinden wird - enttäuscht werden muss. Entsprechend wäre zu erwarten, dass auch bei Absagen ähnliche Strukturen zur Markierung von Dispräferenz auffindbar sind. Dies ist etwa in dem folgenden Beispiel der Fall:

\section{Dialog \#1717}

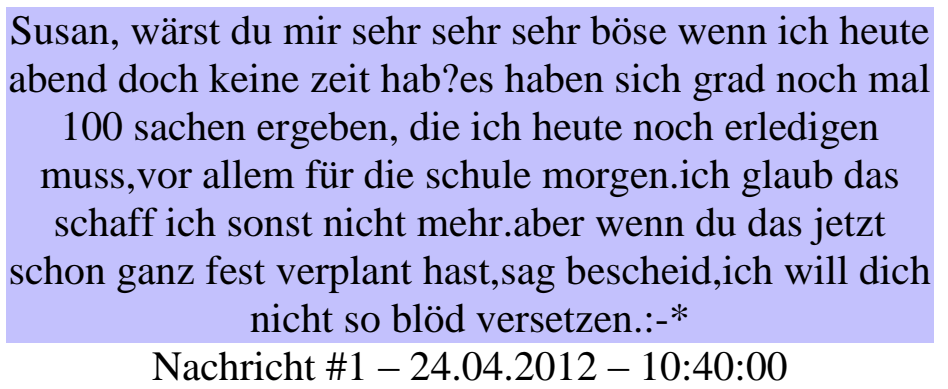

18 Offen bleibt an dieser Stelle, inwieweit Vorschläge von alternativen Terminen als fester Bestandteil von Ablehnungen gewertet werden können. In den untersuchten Daten kommen sie in 12 der 35 Verabredungsablehnungen vor. 
Die Schreiberin, die sich hier per SMS an ihre Freundin Susan wendet, vermeidet eine explizite Verbalisierung einer Absage; stattdessen impliziert sie dies lediglich in ihrer (Suggestiv-)Frage (wärst du mir sehr sehr sehr böse wenn ich heute abend doch keine zeit hab?), die durch die Reduplikation der Partikel "sehr" eine starke affektive Aufladung erfährt. Es folgt eine vergleichbar lange Begründung, warum es die Schreiberin vorziehen würde, das zuvor vereinbarte Treffen abzusagen. Hierdurch wird die Absage als dispräferiert markiert. Auch in dem letzten Teil ihrer Nachricht stellt es die Schreiberin der adressierten Susan zur Disposition, ob sie das Treffen nicht doch stattfinden lassen möchte. Hier orientiert sich die Schreiberin an möglichen negativen Reaktionen Susans, die den Eindruck gewinnen könnte, die Schreiberin würde sie "blöd versetzen". Auch wenn sie durch hyperbolische Formulierungen (sehr sehr sehr böse, noch mal 100 Sachen) ${ }^{19}$ und ihre ausführliche Begründung klar macht, dass sie eine Absage des Treffens vorzieht, überlässt die Schreiberin letztendlich ihrer Freundin Susan die Entscheidung. Die Schreiberin positioniert sich somit als eine Person, der eine explizite Absage nicht zusteht.

Eine solche auf mehreren Ebenen als dispräferiert markierte Absage bildet jedoch nicht den Schwerpunkt der im Korpus gefundenen Absage-SMS. Der überwiegende Teil von Verabredungsabsagen wird in den untersuchten SMS-Dialogen wie im folgenden Beispiel realisiert:

\section{Dialog \#145}

Muss leider absagen. Muss noch nen referat fertig machen. Könnte aber morgen abend 19uhr? Wie siehts da bei dir aus?

Nachricht \#2 - 02.05.2011 - 9:34:05

Der Schreiber beginnt seine Nachricht mit einer expliziten, wenn auch durch die Bedauernsbekundung "leider" modalisierten Absage. Auch hier deutet das Modalverb müssen abermals einen externen Zwang an. Es folgt eine kurze Begründung, in der der Schreiber ausführt, was ihn zu der Absage veranlasst, bevor auch hier im Sinne eines "good news exit" (Maynard 2003) ein alternativer Termin vorgeschlagen wird. Eine explizite Entschuldigungsfloskel findet sich in der gegebenen Nachricht nicht.

Auch wenn sich in diesen beiden Absage-SMS in Bezug auf ihre Direktheit unterschiedliche Strategien der sprachlichen Realisierung einer Absage ausmachen lassen, so stimmen die Nachrichten jedoch in ihrem internen sequentiellen Aufbau miteinander überein: Zunächst wird die Absage realisiert bzw. wie im Fall von Dialog \#1717 angedeutet und erst dann erfolgt die Begründung, warum es zu der Absage eines zuvor vereinbarten Termins kommen muss. Hierin unterscheiden sich die meisten Absage-SMS von dem überwiegenden Teil von Verabredungsablehnungen (zu einer Ausnahme siehe Abschnitt 4.4). Im Folgenden soll das für Absage-SMS am häufigsten vorkommende Muster genauer untersucht werden, bevor in einem zweiten Schritt die Bedeutung der sequentiellen Einbindung der Absage-SMS in einen SMSDialog für dieses Muster reflektiert wird.

\footnotetext{
${ }^{19}$ Cf. Pomerantz (1986) zur legitimierenden Funktion von extreme case formulations.
} 


\subsection{Muster: Absage vor Begründung}

Anhand des folgenden Beispiels sollen prototypische Eigenschaften des dominanten Musters von Absage-SMS in den untersuchten Daten dargestellt werden. Der fünfzügige Dialog \#951 beginnt mit einer Absage von Schreiberin A.

\section{Dialog \#951}

Hey liebe emma, muss dir jetzt leider doch für mensa morgen absagen, muss in sonne blöde sprechstunde, sorry! Nächste woche wieder? Drücka! Nachricht \#1 - 10.05.2011 - 18:44:41

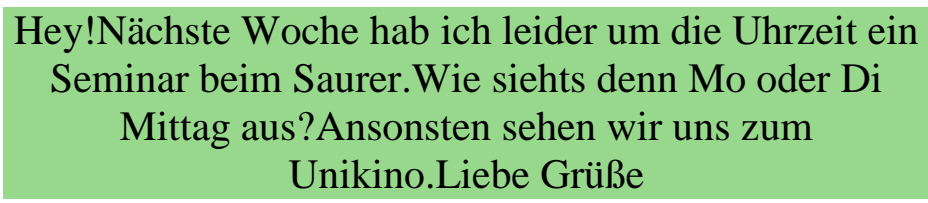

Nachricht \#2 - 10.05.2011 - 18:45:00

Am mo könnt ich um 12.15 und am di um 12. Passt das

für dich? Liebe grüße!

Nachricht \#3 - 10.05.2011 - 18:51:56

Dienstag um 12 wäre super!Dann wünsch ich dir noch ne schöne Woche!LG

Nachricht \#4 - 01.01.2011 - 18:53:10

Alles klar! Dir auch :-)!

Nachricht \#5 - 10.05.2011 - 18:56:43

Sekundenangaben exakt

In Nachricht \#1 initiiert Schreiberin A einen SMS-Dialog, in dem sie einen zuvor vereinbarten Termin absagt. Nach dem Einstieg mit einem Gruß und einer namentlichen Anrede schließt sich direkt die eigentliche Absage an (muss dir jetzt leider doch für mensa morgen absagen). Der Zeitpunkt der abgesagten Verabredung wird hier also - im Gegensatz zu der Nichtnennung in den meisten Ablehnungen von Verabredungsvorschlägen (cf. Abschnitt 4) - explizit genannt. Wie schon in Dialog \#145 ist die Absage auch hier mit einer Bedauernsbekundung (leider) und dem Modalverb müssen realisiert, mit dem die Schreiberin anzeigt, nicht anders handeln zu können. Auch die folgende Begründung ist durch das Modalverb müssen als Zwang gekennzeichnet: muss in sonne blöde sprechstunde. Die Schreiberin A übernimmt somit nicht die volle Verantwortung für die Absage. An dieser Stelle schließt sich nun eine explizite, wenn auch kurz gehaltene Entschuldigungsfloskel an (sorry!), die u. a. durch den Sprachwechsel Informalität bzw. Nähe kontextualisiert. Ebenso wie bei den Verabredungsablehnungen finden sich auch in Absage-SMS z. T. Verweise auf kommende Treffen (in der hier zitierten AbsageSMS als Nächste woche wieder? realisiert), die ebenfalls als "good news exit" (Maynard 2003) gewertet werden können.

Im Folgezug \#2 geht die angeschriebene Emma nicht auf die eigentliche Absage von Schreiberin A ein, sondern realisiert eine Verabredungsablehnung im Hinblick auf den von Schreiberin A vorgeschlagenen Alternativtermin, bevor sie wiederum selbst alternative Terminvorschläge unterbreitet, von denen in der Folge einer ausgewählt (Nachricht \#4) und als 
neue Verabredung ratifiziert wird (Nachricht \#5). Die von Schreiberin A übermittelte AbsageSMS ist hier also der erste Zug eines insgesamt fünfzügigen SMS-Dialogs.

\subsection{Zur dialogischen Einbindung von SMS-Absagen}

Nicht alle Absage-SMS bilden in den untersuchten Daten den ersten Zug eines Dialogs; in neun von 28 SMS-Absagen gehen der eigentlichen Absage-SMS weitere Züge voraus. Für diese Fälle lassen sich zwei prototypische sequentielle Kontexte beschreiben, in denen die AbsageSMS nicht an erster Position verbalisiert wird.

Zum einen können Absagen eines vorher vereinbarten Termins als zweiter Zug eines SMSDialogs realisiert sein. In diesem Fall ist der vorhergehende erste Zug meist eine Erinnerung an eine vorher getroffene Verabredung. In dem folgenden Beispiel schreibt Schreiberin A ihrem Bruder, um ihn an eine Verabredung zum Grillen bei ihren Eltern zu erinnern.

\section{Dialog \#1022}

Na wo bleibt ihr denn?papa hat grill grad angemacht Nachricht \#1 - 14.05.2011 - 16:36:01

Wir kommen nicht mehr.. Die sind noch am pennen und schaffs auch noch hoch. War lang gestern. Sry

Nachricht \#2 - 14.05.2011 - 16:41:01

\section{Traurig! Hab mich schon gefreut}

Nachricht \#3 - 14.05.2011 - 16:44:01

\section{Sekundenangaben fiktiv}

Die von Schreiber B zu Beginn von Nachricht \#2 gegebene Mitteilung Wir kommen nicht mehr.. kann als Absage des vereinbarten Grilltermins gewertet werden. Dem in Abschnitt 5.2 beschriebenen Muster von Absage-SMS entsprechend schließt sich nun eine Begründung an, in der der Bruder mitteilt, dass sowohl Menschen in seinem Umfeld (Die) als auch er selbst noch schlafen oder nicht recht aufstehen können, da sich die Gruppe am Abend zuvor wohl erst spät zu Bett begeben hat (War lang gestern.). Eine explizite Entschuldigungsfloskel (in der Kurzform Sry für sorry realisiert) wird ebenfalls erst nach der Absage und der Begründung gegeben. Es zeigt sich also, dass das prototypische Muster für Absage-SMS auch bei einer dialogischen Einbindung von Absagen als zweitem Zug beibehalten wird. Die Nachricht ist jedoch insofern als zweiter Zug eines Dialogs markiert, als eine Anrede hier (anders als etwa in Dialog \#951) ausbleibt und auch der Verabredungszeitpunkt nicht noch einmal verbalisiert wird (ähnlich wie auch schon die Absage aus Dialog \#145, die ebenfalls als Reaktion auf eine Terminerinnerung folgt). Auch dieser Befund deutet darauf hin, dass sich die interne Struktur von Absage-SMS bereits in hohem Maße verfestigt hat. Der Dialog wird durch eine Reaktion von Schreiberin A auf die Absage fortgeführt, in der sie ihr Bedauern über das Nichtzustandekommen des eigentlich anvisierten Treffens zum Ausdruck bringt.

Eine andere Art der dialogischen Einbindung von Absage-SMS soll anhand des folgenden Beispiels beschrieben werden. Absagen sind in den untersuchten Daten auch dann nicht in initialer Position eines SMS-Dialogs zu finden, wenn sich die SchreiberInnen zuvor per SMS verabredet haben. 


\section{Dialog \#882}

Hey, hast du lust heute abend noch was trinken zu gehen? Wir können ja versuchen den rest der "germanistik-truppe" auch zusammen zu trommeln.. Viele grüße jonas

Nachricht \#1 - 12.10.2010 - 15:29:59

Hey!Gerne.Dann werd ich Julia gleich mal im Seminar fragen. Wo und wann wollen wir uns denn treffen?20h vorm Institut?Liebe Grüße,Caro

Nachricht \#2 - 12.10.2010 - 15:32:54

Ja, klingt gut. Aber überlegt euch schon mal ne bar. Ich hab nämlich noch keinen plan hier:-D

Nachricht \#3 - 12.10.2010 - 15:43:41

Hey Jonas!Ich muss mich für heute Abend leider abmelden.Hab seit $2,5 \mathrm{~h}$ wahnsinnige

Kopfschmerzen.:0/Lass uns das Losgehen doch nächste Woche nachholen!LG,Caro

Nachricht \#4 - 12.10.2010 - 18:45:17

Ja kein ding. Warst du denn auch nicht beim seminar?

Nachricht \#5 - 12.10.2010 - 19:03:19

Sekundenangaben exakt

Jonas' initiale Verabredungseinladung (Nachricht \#1) wird von der angeschriebenen Caro zunächst angenommen, bevor sie dann selbst eine Uhrzeit und einen Treffpunkt vorschlägt (Nachricht \#2). Dieser Vorschlag wiederum wird von Jonas ratifiziert und mit der Bitte an Caro versehen, den weiteren Verlauf der Verabredung zu planen (Nachricht \#3), auf die Caro jedoch nicht weiter eingeht. Mit drei Stunden Abstand schließt sich eine Absage Caros in Nachricht \#4 an, die wiederum in dem prototypischen Muster von Absage-SMS (Anrede, Absage mit Nennung des eigentlich verabredeten Zeitpunkts, Begründung) realisiert ist und dem sich ein alternativer Terminvorschlag als "good news exit" (Maynard 2003) anschließt. Im folgenden Zug stimmt Jonas zunächst der vorgeschlagenen Terminalternative zu ("Ja"), um dann durch einen entlastenden Kommentar (kein ding) auf die Absage zu reagieren (cf. Maynard (2003: 177-179) zu "remedy announcements").

Die so in den Dialog eingebettete Absage-SMS ist jedoch nicht nur in der zeitlichen Aufeinanderfolge der Nachrichten vom ersten Teil des Dialogs abgesetzt: Eine Fortführungserwartung an Caro ist lediglich durch die an sie gerichtete Bitte der Weiterplanung des Abends gegeben. Die Absage-SMS ist jedoch durch die vorherigen Nachrichten \#1 bis \#3 nicht konditionell relevant gemacht worden. Auch die explizite Anrede Hey Jonas! (Nachricht \#4) ist für eine Nachricht inmitten eines Dialogs als abweichend einzustufen (cf. Günthner 2011). Hier kann sich also die Frage anschließen, inwieweit bei dem vorliegenden Beispiel noch von einem oder schon von zwei SMS-Dialogen ausgegangen werden kann. Die Person, die die oben gegebene Abfolge von Nachrichten in die SMS-Datenbank eingespeist hat, kann beispielsweise den thematischen Zusammenhang als Indikator für die Zugehörigkeit zu einem Dialog gewertet haben. Zieht man jedoch den zeitlichen Abstand von drei Stunden sowie die 
explizite Anrede als Indikatoren für den Beginn eines neuen Dialogs heran, so kann dafür argumentiert werden, dass die Absage-SMS ein erstes Paarteil aus einem Adjazenzpaar "Absage - Bestätigung/Bewertung der Absage" darstellt (siehe Abschnitt 5.3).

\subsection{Zwischenfazit zu SMS-Absagen}

Absage-SMS unterscheiden sich von Verabredungsablehnungen per SMS zum einen in ihrem internen sequentiellen Aufbau. Wird die sprachliche Handlung der Ablehnung bei Verabredungsablehnungen dadurch als dispräferiert markiert, dass sie "verspätet" nach einer (teilweise ausführlichen) Begründung positioniert oder überhaupt nicht explizit verbalisiert wird, so wird die eigentliche Mitteilung der Absage in Absage-SMS nach einer Begrüßung (meist Gruß und Namensnennung zusammen) oder direkt zu Beginn der Nachricht platziert. Explizite Entschuldigungsfloskeln kommen nicht in allen der untersuchten Nachrichten vor. In den Fällen, in denen sie realisiert werden, finden sie sich in der Regel in der Position nach der Begründung. In Absage-SMS kommt expliziten Entschuldigungsfloskeln anders als in Verabredungsablehnungen nicht die Rolle eines Kontextualisierungshinweises für das Misslingen einer Terminkoordination zu.

Zum anderen sind Absagen von Verabredungsablehnungen auch in ihrer sequentiellen Einbindung in einen SMS-Dialog voneinander zu unterscheiden. Während aus der Definition von Verabredungsablehnungen bei Kiesendahl (2011) hervorgeht, dass sie eine reaktive sprachliche Handlung darstellen, finden sich in bisherigen linguistischen Arbeiten widersprüchliche Aussagen zum sequentiellen Status von Absagen (cf. Abschnitt 3). Auch wenn die untersuchten Absage-SMS nicht in allen Fällen einen SMS-Dialog initiieren (Ausnahme: als Reaktion auf eine Terminerinnerung, Dialog \#1022), so kann doch festgehalten werden, dass Absagen in den meisten Fällen nicht durch vorhergehende Nachrichten relevant gemacht werden. In Abgleich zu dem Befund, dass Verabredungsablehnungen häufig ohne Reaktion bleiben, werden Absage-SMS in der Regel durch die jeweiligen DialogpartnerInnen bestätigt oder kommentiert. In den auf Absage-SMS folgenden Zügen findet sich häufig eine Bewertung (Traurig!, Dialog \#1022) oder Bestätigung der Absage (Alles klar, Dialog \#1085, hier jedoch nicht genauer analysiert), ebenso wie Kommentare, die die absagende Person entlasten (kein ding, Dialog \#882). Reaktionen wie in Dialog \#951, in dem die Adressatin der Absage lediglich auf einen vorgeschlagenen Alternativtermin eingeht, bilden in den untersuchten Daten die Ausnahme. In diesem Sinne soll dafür argumentiert werden, dass Absagen eines vorher vereinbarten Termins per SMS mit Ausnahme der Reaktionen auf eine Terminerinnerung den ersten Paarteil des Adjazenzpaares "Absagen - Bestätigung/Bewertung der Absage" bilden.

Dies kann auch als Erklärung dafür dienen, warum die ebenso wie Verabredungsablehnungen potenziell gesichtsbedrohende sprachliche Handlung der Absage nicht in gleichem Maße als dispräferiert markiert wird. ${ }^{20}$ Zwar können auch die vorhandenen Begründungen sowie die Bedauernsbekundungen (leider) und die seltener vorkommenden Entschuldigungsfloskeln als Marker für die Dispräferenz von Absagen gewertet werden, jedoch werden diese Elemente anders als bei Verabredungsablehnungen per SMS prototypischerweise nicht genutzt, um die

\footnotetext{
${ }^{20}$ Dass nicht nur reaktive, sondern auch initiale Handlungen als dispräferiert markiert sein können, illustriert etwa Davidson (1984) (cf. auch Pomerantz/Heritage 2013).
} 
eigentliche Mitteilung der Absage hinauszuzögern. Zur Initiierung einer neuen sprachlichen Handlung, die auf einen vorherigen Interaktionskontext zurückverweist, muss diese Anbindung an die vorherige Verabredung zunächst noch einmal wiederhergestellt werden. ${ }^{21}$ Dies spiegelt sich etwa auch darin, dass in den Absage-SMS in der Regel noch einmal der Zeitpunkt des Treffens genannt wird (cf. heute Abend, Nachricht \#1 in Dialog \#1717; für mensa morgen, Nachricht 1 in Dialog \#951; heute Abend Nachricht \#4 in Dialog \#882).

\section{Diskussion: Präferenzorganisation in SMS-Dialogen}

Auch wenn in den vorhergehenden Analysen gezeigt werden konnte, dass gewisse für mündliche Interaktionen bereits umfassend beschriebene Verfahren der Markierung von Dispräferenz (cf. Pomerantz/Heritage 2013) auch in den untersuchten SMS-Dialogen auffindbar sind, so müssen jedoch auch Unterschiede zur Präferenzorganisation mündlicher Interaktionen festgehalten werden. Ein in Gesprächen zentrales Mittel, um etwa ein zweites Paarteil als dispräferiert zu markieren, besteht darin, einen zeitlichen "Bruch" zwischen dem ersten und dem zweiten Paarteil herzustellen (cf. Schegloff 2007: 67). Dies kann zum einen dadurch realisiert werden, dass der zweite Paarteil durch vorangestellte Begründungen "verzögert" realisiert wird (cf. Pomerantz 1984 zu "delay"), wie dies in den untersuchten SMSDialogen etwa beim ersten Muster der Verabredungsablehnungen der Fall ist (cf. Abschnitt 4.1).

Zum anderen kann Dispräferenz eines zweiten Paarteils auch durch eine minimale Pause zum vorhergehenden Gesprächsbeitrag angezeigt werden ("inter-turn gap", Schegloff 2007: 67). Bei der Übertragung dieses Verfahrens aus der mündlichen Interaktion auf SMS-Dialoge bestehen Schwierigkeiten nicht nur darin, dass Unklarheit über einen "relevanten" zeitlichen Abstand von SMS-Nachrichten herrschen kann, ab wann also eine nicht erfolgte Antwort als "verzögert" gelten kann (cf. etwa Günthner/Kriese (2012) zu unterschiedlichen Schweigetoleranzen in deutschen und chinesischen SMS-Dialogen). Das Ausbleiben einer zeitlich direkt folgenden Reaktion wird in keiner der hier untersuchten Verabredungsablehnung und Absagen als relevantes "Schweigen" behandelt, das eine Ablehnung oder Absage impliziert (anders als dies etwa Laursen (2005) bei einer Gruppe jugendlicher SMS-NutzerInnen feststellt). Während in manchen Dialogen nur wenige Minuten zwischen dem Verabredungsvorschlag und der Ablehnung liegen (etwa in den Dialogen \#528 und \#295), können jedoch auch wie in Dialog \#456 etwa 40 Minuten vergehen, bevor eine Antwort auf einen Verabredungsvorschlag folgt ohne dass die Nichtantwort vorher von der anfragenden Person noch einmal thematisiert worden ist. ${ }^{22}$

Ebenso zeigen sich Unterschiede in den Funktionen, die solche Verzögerungen jeweils in mündlichen Interaktionen und in SMS-Dialogen haben: In mündlichen Interaktionen führen zeitliche Verzögerungen des Beginns oder der Platzierung von zweiten Paarteilen häufig dazu,

\footnotetext{
${ }^{21}$ Auch Schegloff (1988: 445) weist darauf hin, dass Andeutungen einer dispräferierten Handlung nur in solchen Kontexten platziert werden können, in denen SprecherInnen davon ausgehen können, dass ihre Andeutungen vom Gegenüber auch verstanden werden können.

22 Lediglich in dem SMS-Streitdialog \#1647 (Abschnitt 4.4) wird das Schweigen der adressierten Person durch Schreiberin A als ein relevantes Ausbleiben einer Reaktion interpretiert, jedoch nicht in Zusammenhang mit der in Nachricht \#7 erfolgten Ablehnung des Verabredungsvorschlags.
}

ISSN 1615-3014 
dass die so als dispräferiert markierte sprachliche Handlung gar nicht mehr expliziert werden muss (weil etwa die Person, die zuvor eine Einladung ausgesprochen hat, diese nach Zögerungen ihres Gegenübers - teilweise auch schon in Überlappung - zurücknimmt oder so umformuliert, dass das Gegenüber die Einladung "unproblematisch" ablehnen kann; cf. Davidson 1984). ${ }^{23}$ Somit erweist sich der sequentielle Auf- und Ausbau von Gesprächen, bei dem Produktion und Rezeption von Gesprächsbeiträgen synchron verlaufen, als eine Grundbedingung, für Möglichkeiten des "Gegensteuerns" bei potenziellen Ablehnungen, aber auch bei schrittweise eingeführten und damit als dispräferiert markierten ersten Paarteilen.

In den hier untersuchten SMS-Dialogen werden jedoch Verabredungsablehnungen und Absagen nicht Zug für Zug vollzogen, sodass das Gegenüber für eine Umformulierung oder Suspendierung der Einladung "gegensteuern" könnte. Prinzipiell denkbar wäre etwa, zunächst Nachrichten zu senden, die nur "reluctance marker" (Bilmes 1988) wie etwa das "Ehm" aus Dialog \#528 enthalten. Stattdessen werden jedoch alle oben herausgearbeiteten Komponenten von initialen Absagen und reaktiven Ablehnungen (Mitteilung der Absage bzw. Ablehnung, Begründungen, mitunter auch explizite Entschuldigungen oder Vorschläge von Alternativterminen) in nur einer Nachricht übermittelt, die ein direktes Umsteuern des Dialogpartners/der Dialogpartnerin ausschließen. Für das Mündliche beschriebene Verzögerungsstrukturen wie vorangestellte Verzögerungssignale (siehe auch Davidson 1984: 103), auf die die adressierte Person direkt reagieren kann, finden sich in den untersuchten Daten nicht. Hierin zeigt sich, dass eine direkte Übertragung der Prinzipien von mündlichen Interaktionen auf schriftlich realisierte Dialogstrukturen nicht möglich ist (cf. auch Günthner $(2011$; 2012) zu Unterschieden in der sequentiellen Organisation von SMS-Dialogen und face-to-face-Interaktionen). Die Markierung von Dispräferenz in der schriftbasierten, asynchronen bzw. quasi-synchronen SMS-Kommunikation ist in den untersuchten Daten also dadurch bestimmt, dass Produktion und Rezeption zeitlich voneinander getrennt stattfinden. Inwieweit sich diese Befunde auch in neueren Kommunikationsformen wie "WhatsApp", innerhalb derer einzelne Beiträge einem Chat ähnlich potenziell schneller aufeinander folgen können, replizieren lassen oder ob sich hier Nachrichten, die lediglich Verzögerungssignale enthalten, finden, muss in zukünftigen Untersuchungen noch gezeigt werden.

\section{$7 \quad$ Zur Dialogizität von Absagen und Verabredungsablehnungen}

Das Handy und die mit ihm verbundene Kommunikationsform der SMS haben sich längst als wichtiges Instrument der Koordination sozialer Kontakte etabliert. Man erreicht Freunde und Bekannte zwecks Terminabsprachen zu (fast) jeder Zeit und an (fast) jedem Ort. Eine abschließende und verbindliche Planung eines Treffens ist dank des Mobiltelefons nicht mehr im gleichen Maße notwendig, wie dies etwa noch vor einigen Jahren der Fall war. Termine können im Sinne von Lings Konzept der "micro-coordination" (Ling 2008; cf. auch Ling/Baron 2013) kurzfristig geplant, verschoben oder sogar abgesagt werden. Auch an dem hier untersuchten Korpus ist ablesbar, dass Verabredungen nicht mehr lang im Voraus, sondern in der Regel sehr kurzfristig per SMS koordiniert werden: Die meisten SMS-Dialoge befassen sich mit der Abstimmung von Terminen am gleichen Tag.

\footnotetext{
${ }^{23}$ Siehe auch Pomerantz (1984: 77): "In general, dispreferred-action turn organization serves as a resource to avoid or reduce the occurrences of overtly stated instances of an action."
}

ISSN 1615-3014 
Wenn Verabredungen in zunehmendem Maße per SMS vereinbart werden, so verwundert es nicht, dass auch Absagen per SMS verschickt werden. Da Ablehnungen und Absagen die bisherige Terminkoordination misslingen lassen, können sie als potenziell gesichtsbedrohende Aktivitäten eingestuft werden. Auch wenn Stilratgeber darauf hinweisen, dass Absagen bzw. schlechte Nachrichten am besten mündlich übermittelt werden sollen, zeigen die hier untersuchten SMS-Dialoge, dass es nicht unüblich ist, Verabredungsvorschläge per SMS abzulehnen oder einen Termin per SMS abzusagen. An den hier durchgeführten Analysen des sequentiellen Aufbaus der Ablehnungs- bzw. Absage-SMS konnte festgestellt werden, dass sich diese soziale Norm in einer musterhaften Markierung der Dispräferenz von Ablehnungsund Absage-SMS niederschlägt. Hier ist jedoch auch die jeweilige sequentielle Einbindung in einen SMS-Dialog mit in den Blick zu nehmen. Muss ein Schreiber/eine Schreiberin auf einen Verabredungsvorschlag reaktiv Bezug nehmen, so wird die explizite Formulierung der Verabredungsablehnung dadurch als dispräferiert markiert, dass sie erst nach einer Begründung realisiert oder lediglich angedeutet wird. Ein expliziter Bezug zu dem vorgeschlagenen Termin wird nicht noch einmal verbalisiert. Die antwortende Person geht in der sequentiellen und sprachlichen Gestaltung ihrer Ablehnungs-SMS davon aus, dass die Person, die den Verabredungsvorschlag unterbreitet hat, die zum Verstehen ihres zweiten Zuges benötigten Informationen aus dem bisherigen Interaktionskontext rekonstruieren kann.

Bei der Gattung der Absage-SMS muss dieser Kontext jedoch erst (wieder) hergestellt werden. Dies geschieht zum einen mit einer expliziten und anfänglichen Formulierung einer Absage, in der zudem in den meisten Fällen der Verabredungszeitpunkt noch einmal angeführt wird. Auch durch die häufig in Absage-SMS vorkommenden Einleitungen mit Gruß und Anrede verdeutlichen die SchreiberInnen, dass sie mit der Absage nicht an einen direkt vorhergehenden Beitrag anknüpfen, sondern in der Regel einen neuen SMS-Dialog initiieren.

Die Herstellung und Aufrechterhaltung von sozialen Beziehungen spielt jedoch auch in solchen Nachrichten, in denen die Terminkoordination misslingt, eine große Rolle. Auch wenn eine Person einen vorher vereinbarten Termin absagen oder einen Verabredungsvorschlag ablehnen muss, betonen die SchreiberInnen immer ihr prinzipielles Interesse an weiteren Kontakten.

\section{Literatur}

Androutsopoulos, Jannis K./Schmidt, Gurly (2004): "löbbe döch. Beziehungskommunikation mit SMS". Gesprächsforschung. Online-Zeitschrift zur verbalen Interaktion 5: 50-71.

Androutsopoulos, Jannis K./Schmidt, Gurly. (2002): "SMS-Kommunikation: Ethnographische Gattungsanalyse am Beispiel einer Kleingruppe". Zeitschrift für Angewandte Linguistik 36: 49-79.

Atkinson, John Maxwell/Drew, Paul (1979): Order in Court. The Organisation of Verbal Interaction in Judicial Settings. London: Macmillan Press.

Bernicot, Josie et al. (2012): "Forms and functions of SMS messages: A study of variations in a corpus written by adolescents". Journal of Pragmatics 44: 1701-1715.

Bilmes, Jack (1988): "The concept of preference in conversation analysis". Language in Society 17/2: 161-181.

Botica, Melania (2012): "Benimmregeln für den Handy-Gebrauch. Telefonieren mit Stil.". http://www.focus.de/schule/familie/medien-tipps/benimmregeln-fuer-den-handy-gebrauchtelefonieren-mit-stil_aid_694677.html, letzter Zugriff im September 2013). 
Brown, Penelope/Levinson, Stephen C. (1987): Politeness. Some Universals in Language Usage. Cambridge: Cambridge University Press.

Buttny, Richard/Morris, G. H. (2001): "Accounting". In: Robinson, W. Peter/Giles, Howard (eds.) (2001): The New Handbook of Language and Social Psychology. Chichester: John Wiley and Sons: 285-301.

Davidson, Judy (1984): "Subsequent versions of invitations, offers, requests, and proposals dealing with potential or actual rejection". In: Atkinson, John Maxwell/Heritage, John (eds.) (1984): Structures of Social Action. Studies in Conversation Analysis. Cambridge: Cambridge University Press: 102-128.

Davies, Bethan L./Merrison, Andrew John /Goddard, Angela (2007): "Institutional apologies in UK higher education: Getting back into the black before going into the red." Journal of Politeness Research 3/1: 39-63.

Dittmann, Jürgen (2006): "Konzeptionelle Mündlichkeit in E-Mail und SMS". In: Reeg, Ulrike (ed.) (2006): Interkultureller Fremdsprachenunterricht: Grundlagen und Perspektiven. Bari: Edizioni di Pagina: 79-97.

Döring, Nicola (2002): "'1x Brot, Wurst, 5 Sack Äpfel I.L.D.' - Kommunikative Funktionen von Kurzmitteilungen". Zeitschrift für Medienpsychologie 14/3: 118-128.

Drescher, Martina (1994): "Für zukünftige Bewerbungen wünschen wir Ihnen mehr Erfolg. Zur Formelhaftigkeit von Absagebriefen". Deutsche Sprache 22/2: 117-137.

Drew, Paul (1984): "Speakers' reportings in invitation sequences." In: Atkinson, J. Maxwell/ Heritage, John (eds.) (1984): Structures of Social Action. Studies in Conversation Analysis. Cambridge: Cambridge University Press: 129-151.

Duden ( $\left.{ }^{5} 2003\right)$ : Deutsches Universalwörterbuch. Mannheim: Dudenverlag.

Dürscheid, Christa (2007): "E-Mail und SMS - ein Vergleich". In: Ziegler, Arne/Dürscheid, Christa (eds.) (2007): Kommunikationsform E-Mail. Tübingen: Stauffenburg Verlag: 91114.

Dürscheid, Christa (2002): "SMS-Schreiben als Gegenstand der Sprachreflexion". Networx 28: $1-16$.

Dürscheid, Christa/Brommer, Sarah (2009): "Getippte Dialoge in neuen Medien. Sprachkritische Aspekte und linguistische Analysen". Linguistik online 37/1: 3-20.

Günthner, Susanne (2012): "'Lupf meinen Slumpf: Die interaktive Organisation von SMSDialogen". In: Ayaß, Ruth/Meyer, Christian (eds.) (2012): Sozialität in Slow Motion. Theoretische und empirische Perspektiven. Wiesbaden: Springer VS: 353-372.

Günthner, Susanne (2011): "Zur Dialogizität von SMS-Nachrichten - eine interaktionale Perspektive auf die SMS-Kommunikation". Networx 60: 1-40.

Günthner, Susanne (1999): "Vorwürfe in der Alltagskommunikation". In: Bergmann, Jörg/Luckmann, Thomas (eds.) (1999): Kommunikative Konstruktion von Moral. Band 1: Struktur und Dynamik der Formen moralischer Kommunikation. Opladen, Wiesbaden: Westdeutscher Verlag: 206-241.

Günthner, Susanne (1995): "Gattungen in der sozialen Praxis. Die Analyse 'kommunikativer Gattungen' als Textsorten mündlicher Kommunikation". Deutsche Sprache 3: 193-218.

Günthner, Susanne/Kriese, Saskia (2012): "Dialogizität in der chinesischen und deutschen SMS-Kommunikation - eine kontrastive Studie". Linguistik online 57/7: 43-70.

Heritage, John (1984): Garfinkel and Ethnomethodology. Cambridge: Polity Press. 
Herring, Susan C./Stein, Dieter/Virtanen, Tuija (2013): "Introduction to the pragmatics of computer-mediated communication". In: Herring, Susan C./Stein, Dieter/Virtanen, Tuija (eds.) (2013): Pragmatics of Computer-Mediated Communication. Berlin: de Gruyter: 3-32.

Imo, Wolfgang (2012): "Fischzüge der Liebe: Liebeskommunikation in deutschen und chinesischen SMS-Sequenzen". Linguistik online 56/6: 21-38.

Jucker, Andreas H./Dürscheid, Christa (2012): "The linguistics of keyboard-to-screen communication. A new terminological framework". Linguistik online 56/6: 39-64.

Kiesendahl, Jana (2013): "Apologies and excuses in academic e-mail communication: Differentiation and characterization from a pragmatic perspective". In: Bieswanger, Markus/Koll-Stobbe, Amei (eds.) (2013): New Approaches to the Analysis of Linguistic Variability. Frankfurt am Main, Lang: 141-164.

Kiesendahl, Jana (2011): Status und Kommunikation. Ein Vergleich von Sprechhandlungen in universitären E-Mails und Sprechstundengesprächen. Berlin: Erich Schmidt Verlag.

König, Katharina/Bahlo, Nils (2014): "SMS, WhatsApp \& Co. - Forschungsstand und Analyseperspektiven". In: König, Katharina/Bahlo, Nils (eds.) (2014): SMS, WhatsApp \& Co. Gattungsanalytische, kontrastive und variationslinguistische Perspektiven zur Analyse mobiler Kommunikation. Münster, Monsenstein und Vannerdat: 1-16.

Kotthoff, Helga (1993): "Disagreement and concession in disputes: On the context-sensitivity of preference structures". Language in Society 22/2: 193-216.

Krause, Melanie/Schwitters, Diana (2002): "SMS-Kommunikation - Inhaltsanalyse eines kommunikativen Phänomens." Networx 27: 1-35.

Laursen, Ditte (2005): "Please reply! The replying norm in adolescent SMS communication". In: Harper, Richard/Palen, Leysia/Taylor, Alex (eds.) (2005): The Inside Text. Social, Cultural and Design Perspectives on SMS. Dodrecht, Springer: 53-73.

Lerner, Gene H. (1996): "Finding 'face' in the preference structure of talk-in-interaction". Social Psychology Quarterly 59/4: 303-321.

Ling, Rich (2008): New Tech, New Ties. How Mobile Communication is Reshaping Social Cohesion. Cambridge: MIT Press.

Ling, Rich (2004): The Mobile Connection. The Cell Phone's Impact on Society. San Francisco: Morgan Kaufmann.

Ling, Rich/Baron, Naomi S. (2013): "Mobile phone communication". In: Herring, Susan C./Stein, Dieter/Virtanen, Tuija (eds.) (2013): Pragmatics of Computer-Mediated Communication. Berlin: de Gruyter: 191-216.

Ling, Rich/Julsrud, Tom/Yttri, Birgitte (2005): "Nascent communication genres within SMS and MMS". In: Harper, Richard/Palen, Leysia/Taylor, Alex (eds.) (2005): The Inside Text. Social, Cultural and Design Perspectives on SMS. Dodrecht: Springer: 75-100.

Maynard, Douglas W. (2003): Bad News, Good News. Conversational Order in Everyday Talk and Clinical Settings. Chicago/London: The University of Chicago Press.

Moraldo, Sandro M. (2002): "Short Message Service (SMS) oder Zur Linguistik der Kurznachrichten". Sprachspiegel 58/5: 155-166.

Nowotny, Andrea (2005): "Daumenbotschaften. Die Bedeutung von Handy und SMS für Jugendliche". Networx 44: 1-45

Pomerantz, Anita (1986): "Extreme case formulations: A way of legitimizing claims". Human Studies 9: 219-229. 
Pomerantz, Anita (1984): "Agreeing and disagreeing with assessments: some features of preferred and dispreferred turn shapes". In: Atkinson, J. Maxwell/Heritage, John (eds.) (1984): Structures of social action: Studies in conversation analysis. Cambridge, Cambridge University Press: 57-101.

Pomerantz, Anita (1978): "Compliment responses. Notes on the co-operation of multiple constraints". In: Schenkein, Jim (ed.) (1978): Studies in the Organization of Conversation. New York: Academic Press: 79-112.

Pomerantz, Anita/Heritage, John (2013): "Preference". In: Sidnell, Jack/Stivers, Tanya (eds.) (2013): The Handbook of Conversation Analysis. Chichester: Blackwell: 210-228.

Pöschl, Sandra/Döring, Nicola (2012): "Access anytime, anywhere, with anyone? Determinanten mobiler Erreichbarkeit in der Handykommunikation - ein Review". In: Marx, Konstanze/Schwarz-Friesel, Monika (eds.) (2012): Sprache und Kommunikation im technischen Zeitalter: Wie viel Internet (v)erträgt unsere Gesellschaft? Berlin, de Gruyter: 279-311.

Robinson, Jeffrey D. (2004): "The sequential organization of 'explicit' apologies in naturally occurring English". Research on Language and Social Interaction 37/3: 291-330.

Schegloff, Emanuel (2007): Sequence Organization in Interaction. A Primer in Conversation Analysis I. Cambridge: Cambridge University Press.

Schegloff, Emanuel (1988): "On an actual virtual servo-mechanism for guessing bad news: A single case conjecture". Social Problems 35/4: 442-457.

Schindler, Kisten (2004): Adressatenorientierung beim Schreiben. Eine linguistische Untersuchung am Beispiel des Verfassens von Spielanleitungen, Bewerbungsbriefen und Absagebriefen. Frankfurt am Main: Peter Lang.

Schlobinski, Peter (2005): "Mündlichkeit und Schriftlichkeit in den Neuen Medien". In: Eichinger, Ludwig/Kallmeyer, Werner (eds.) (2005): Standardvariation. Wie viel Variation verträgt die deutsche Sprache? Berlin, de Gruyter: 126-142.

Schlobinski, Peter/Fortmann, Nadine/Groß, Olivia et al. (2001): "Simsen. Eine Pilotstudie zu sprachlichen und kommunikativen Aspekten in der SMS-Kommunikation." Networx 22: 138.

Schmidt, Gurly (2006): "Sprachliche Variation in der SMS-Kommunikation". In: Schlobinski, Peter (ed.) (2006): Von *hdl* bis *cul8r*. Sprache und Kommunikation in den Neuen Medien. Mannheim: Dudenverlag: 317-333.

Schwitalla, Johannes (2002): "Kleine Botschaften. Telegramm- und SMS-Texte". OBST 64: 33-56.

Scott, Marvin B./Lyman, Stanford M. (1968): "Accounts". American Sociological Review 33/1: $46-62$.

Spagnolli, Anna/Gamberini, Luciano (2007): "Interaction via SMS: Practices of local closeness and reciprocation". British Journal of Social Psychology 46: 343-364.

Spilioti, Tereza (2011): "Beyond genre: Closings and relational work in text messaging". In: Thurlow, Crispin/Mroczek, Kristine (eds.) (2011): Digital Discourse. Language in the New Media. Oxford: Oxford University Press: 67-85.

Staffeldt, Sven (2013): "Auf dem Weg zum pragmatischen Standard mit Entschuldigungen". In: Hagemann, Jörg/Klein, Wolf Peter/Staffeldt, Sven (eds.) (2013): Pragmatischer Standard. Tübingen: Stauffenburg: 85-109. 
Talmy, Leonard (1988): "Force dynamics in language and cognition". Cognitive Science 12: 49-100.

Thurlow, Crispin/Poff, Michele (2013): "Text messaging". In: Herring, Susan C./Stein, Dieter/Virtanen, Tuija (eds.) (2013): Pragmatics of Computer-Mediated Communication. Berlin: de Gruyter: 163-190.

Wagner, Klaus R. (2001): Pragmatik der deutschen Sprache. Frankfurt am Main: Peter Lang. Weidacher, Georg (2011): "Entschuldigungsmails: Konventionalisierung und Variation in der Umsetzung eines Textmusters". In: Luginbühl, Martin/Perrin, Daniel (eds.) (2011): Muster und Variation. Medienlinguistische Perspektiven auf Textproduktion und Text. Bern, Lang: 51-80.

Weinrich, Harald ( $\left.{ }^{3} 2005\right)$ : Textgrammatik der deutschen Sprache. Hildesheim: Georg Olms. 